\section{Prix Nobel de Chimie 2003 \\ Canaux hydriques et ioniques}

Daniel G. Bichet, Lucie Parent, Rémy Sauvé
> Ces prix Nobel récompensent, au travers de la distinction accordée à Peter Agre et Roderick Mackinnon, des avancées décisives dans la connaissance de la structure et du fonctionnement de protéines membranaires, les canaux hydriques et ioniques, essentiels à la communication entre les cellules et leur environnement. <

\section{La découverte des aquaporines}

Peter Agre a 54 ans, c'est un médecin hématologue à I'université Johns Hopkins de Baltimore où il est professeur de chimie biologique et de médecine. Il a découvert «par hasard», comme il se plaît à le dire, les aquaporines. Ces protéines, insérées dans la double couche lipidique de nombreuses membranes cellulaires, confèrent à la membrane où elles sont insérées une perméabilité considérable à l'eau: 3 milliards de molécules $(\rightarrow) \mathrm{m} / \mathrm{s}$ 2001, n ${ }^{\circ} 1$, p. 115 d'eau par seconde, et par monomère d'aquaporine $(\rightarrow)$. Le passage d'eau y est sélectif : l'eau passe, mais pas les protons. Pour les néphrologues, l'insertion ou non de canaux hydriques dans les différents épithéliums du tubule rénal explique leur perméabilité ou leur imperméabilité à l'eau. Ainsi, la branche descendante de l'anse de Henle est très perméable, car l'aquaporine 1 y est insérée. La branche ascendante de l'anse de Henle, imperméable à l'eau et très perméable aux solutés, n'exprime aucune aquaporine.

À la fin des années 1980, Peter Agre ne cherchait pas des aquaporines mais travaillait à l'identification des déterminants moléculaires du groupe Rhésus des globules rouges. À partir de grandes quantités de globules rouges, il isola une fraction membranaire présente en abondance, à partir de laquelle il réussit un séquençage peptidique partiel. Celui-ci permit la construction de sondes génomiques suffisantes pour cribler une banque érythrocytaire et isoler un gène codant pour une protéine membranaire n'appartenant à aucune famille connue. L'expression de cette protéine dans des œufs de xénope révéla une perméabilité à l'eau signifi-

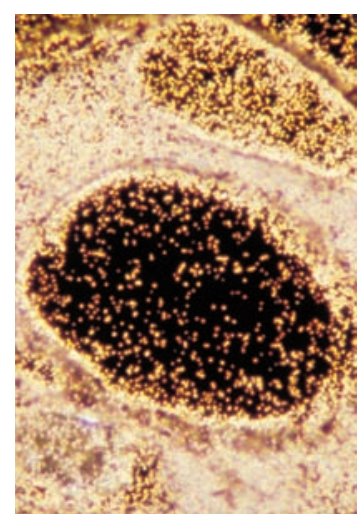

D.G. Bichet : Groupe d'étude des protéines membranaires, Université de Montréal, Chaire de recherche du Canada en génétique des maladies rénales, Service Néphrologie-Génétique, Hôpital du Sacré-Cœur de Montréal, 5400 , boulevard Gouin ouest, Montréal, Québec, H4J 1C5, Canada.

d-binette@crhsc.umontreal.ca

L. Parent, R. Sauvé :

Groupe de recherche en transport membranaire, Département de Physiologie, Université de Montréal,

cativement accrue. Les canaux à eau, dont l'existence était sugCP 6128, Succursalle Centre-ville, Montréal, Québec, H3C 3J7, Canada. gérée depuis de très nombreuses années, étaient ainsi identifiés. La famille de ces canaux allait vite s'agrandir avec plus de dix variétés, des canaux à eau «purs», ne transportant que de l'eau, puis des canaux moins orthodoxes, transportant du glycérol ou d'autres petites molécules. Ces canaux à eau sont présents dans tous les épithéliums perméables de tous les organes et de nombreuses espèces animales et végétales. Leur perte de fonction est responsable de quelques syndromes héréditaires rares (diabètes insipides néphrogéniques autosomiques dominants et récessifs - AQP2, cataracte congénitale - AQP0). Peter Agre a soulevé les pierres de la connaissance dans un ruisseau limpide, l'eau passe dans les membranes modélisées de Bert de Groot (http://www.mpibpc.gwdg.de/abteilungen/071/bgroot /presentations/aqpl_dyn/aqpl_system_movie.html). Si vous êtes un chercheur enthousiaste, il y a d'autres pierres à retourner sur des ruisseaux ou des chemins qui conduisent à Stockholm. $\diamond$

\section{Daniel G. Bichet}




\section{La structure des canaux potassiques}

Roderick MacKinnon, 47 ans, obtint un doctorat en médecine en 1982 à la Tufts Medical School de Boston (MA, USA). Il est professeur de neurobiologie moléculaire et de biophysique à la Rockefeller University à New York (NY, USA). L'attribution du prix Nobel vient couronner des découvertes majeures sur la structure des canaux ioniques spécialisés dans le transport passif des ions. Les canaux ioniques jouent un rôle essentiel, principalement dans le bon fonctionnement des cellules du système nerveux et des muscles squelettiques ou cardiaques. Ce qu'on appelle le potentiel d'action des cellules nerveuses et musculaires apparaît quand un canal ionique situé à la surface de ces cellules est ouvert par un signal chimique envoyé par un neurone adjacent; une impulsion électrique se propage alors grâce à l'ouverture et à la fermeture d'une série de canaux ioniques distincts, cela en l'espace de quelques millisecondes. Structure et fonction des canaux ioniques sont demeurées longtemps méconnues, en raison de leur faible densité et du fait que ce sont des protéines membranaires intrinsèques se prêtant mal à la purification. L'identification des ces protéines a débuté par les travaux de A.L. Hodgkin et A.F. Huxley (prix Nobel de physiologie/médecine, 1963) qui publiaient en 1952 la première caractérisation des mécanismes ioniques de l'excitation des cellules neuronales. En effet, même si le rôle des ions $\mathrm{Na}^{+}, \mathrm{Ca}^{2+}$ et $\mathrm{K}^{+}$dans les propriétés électriques des cellules eucaryotes était connu depuis le début du xxesiècle, il manquait les outils biophysiques et électrophysiologiques par lesquels il est possible de contrôler le potentiel membranaire pour mesurer les courants ioniques produits par chacun de ces ions à travers les membranes cellulaires. Après avoir affiné la méthode du potentiel imposé, Hodgkin et Huxley ont élaboré un modèle de conduction dans l'axone du calmar géant qui prévoyait des chemins de conductance séparés pour les ions $\mathrm{Na}^{+}$et $\mathrm{K}^{+}$. II fallut cependant attendre plus de 30 ans, avec l'arrivée de la méthode du patch clamp mise au point par l'équipe de Bert Sakmann et Erwin Neher (Prix Nobel de physiologie/médecine, 1991) $(\rightarrow)$, pour confirmer que les courants ioniques dans les cellules excitables sont produits lors de l'ouverture et de la fermeture de protéines-pores qui laissent spécifiquement passer des ions. Avec le déploiement spectaculaire des techniques de clonage et de biologie moléculaire dans les années 1990, les structures primaires de nombreux canaux ioniques ont été identifiées grâce aux travaux pionniers des groupes de Shosaku Numa au Japon et Lily Jan aux États-Unis. À l'époque, on se limitait à estimer les structures secondaires de ces protéines à partir d'algorithmes dérivés des méthodes de chimie théorique; car la structure tridimensionnelle de ces protéines membranaires semblait alors inaccessible. Seules les structures de quelques portions cytoplasmiques des canaux ioniques ont été obtenues par résonance magnétique nucléaire (RMN). Roderick Mackinnon provoqua donc la surprise dans l'ensemble de la communauté scientifique lorsqu'il réussit, avec son équipe de recherche, à déterminer la structure spatiale d'un canal sélectif aux ions potassiques en 1998. Grâce à sa contribution, nous pouvons aujourd'hui «voir» des ions passer par les canaux qui s'ouvrent et se referment en réponse à des signaux cellulaires. Ces travaux ont permis d'expliquer pour la première fois, à l'échelle atomique, pourquoi les canaux $\mathrm{K}^{+}$ peuvent si bien discriminer les ions $\mathrm{K}^{+}$des autres ions et distinguer les processus de diffusion des ions dans un canal $(\rightarrow)$.

De plus, en cristallisant des canaux ioniques sous deux états cinétiques distincts (ouvert ou fermé), R. Mackinnon parvint à mettre en évidence les changements de structure qui accompagnent l'ouverture des canaux ioniques. Plus tôt cette année, R. Mackinnon a finalement publié la structure cristalline d'un canal ionique plus complexe, qui s'ouvre en réponse aux changements de potentiel et a encore une fois étonné la communauté scientifique par l'audace des modèles d'activation des canaux ioniques qu'il propose. Le groupe de Mackinnon a aussi élucidé la structure tridimensionnelle des sousunités auxiliaires $\beta$, ainsi que leurs sites d'ancrage sur les canaux potassiques. Avec toutes ces structures cristallines auxquelles s'ajoutent les structures des canaux $\mathrm{K}^{+}$activés par les protéines $\mathrm{G}$ et les canaux sélectifs aux anions, il est maintenant possible de déterminer à l'échelle atomique les sites d'interaction des médicaments, et par conséquent de dessiner avec plus de précision les stratégies thérapeutiques applicables à de nombreuses maladies telles que les arythmies cardiaques, le diabète ou l'épilepsie. Le prix Nobel de chimie de cette année illustre la façon dont la biochimie atteint l'échelle atomique et permet d'élucider des mécanismes subtils de la vie. $\diamond$

\section{Lucie Parent, Rémy Sauvé}

$(\rightarrow) \mathrm{m} / \mathrm{s}$ 2002, n 5 , p. 605

$(\rightarrow) \mathrm{m} / \mathrm{s}$ 1991, nº, p. 985 